\title{
Is Lidocaine better than Magnesium Sulfate as an adjuvant with propofol in Colonoscopy?
}

\author{
Ahmed M. Semeda, Ibrahim A. Yousef and Al Shimaa I. Roushdy \\ Department of Anesthesia, El-Minia Faculty of Medicine
}

\begin{abstract}
Background: Colonoscopy is one of the most commonly performed outpatient procedures throughout the world as a screening, diagnostic, and therapeutic tool. propofol is the most common drug used for sedation during endoscopy either alone or in combination with another agent. Patients and Methods: A total of 90 patients of both gender aged between 18 and 70 years with American Society of Anesthesiologists grade I and II patients scheduled to undergo colonoscopy, under sedation were included in the study. Patients were randomly allocated into 3 equal groups each containing 30 patient. Group (L) received $1.5 \mathrm{mg} / \mathrm{kg}$ of lidocaine IV before induction by propofol and continuous infusion of lidocaine $4 \mathrm{mg} \mathrm{kg} / \mathrm{h}$ after induction, group $(\mathrm{M})$ received50 $\mathrm{mg} / \mathrm{kg}$ of magnesium sulphate before induction by propofol and continuous infusion of magnesium $8 \mathrm{mg} / \mathrm{kg} / \mathrm{h}$ after induction and group (O) as control group received normal saline $0.9 \%$ Sodium Chloride. Results: There was a significant difference in intraoperative hemodynamic stability, total amount of propofol, post procedural pain and analgesic demand, and patient between three groups. Conclusion: we concluded that lidocaine infusion by dose $1.5 \mathrm{mg} / \mathrm{kg}$ loading and $4 \mathrm{mg} / \mathrm{kg} / \mathrm{h}$ as maintenance is more effective than magnesium sulfate by dose $50 \mathrm{mg} / \mathrm{kg}$ loading and $8 \mathrm{mg} / \mathrm{kg} / \mathrm{h}$ maintenance in decreasing total amount of propofol, post procedural pain and analgesic demand, and improving patient satisfaction in patient undergoing colonoscopy under sedation with propofol.
\end{abstract}

Keywords: PSA, colonoscopy, lidocaine, magnesium.

\section{Introduction}

Procedural sedation and analgesia (PSA) is the clinical practice of using pharmacologic agents to achieve a measurable level of sedation while performing typically painful or anxiety-provoking procedures ${ }^{(1)}$.

Colonoscopy is one of the most commonly performed outpatient procedures throughout the world as a screening, diagnostic, and therapeutic tool to evaluate the large intestine from the distal rectum to the cecum $^{(2)}$.

The most common complications in gastrointestinal endoscopy are not related to the procedure, but are related to sedation; they include cardio-respiratory adverse events such as hypoxemia, hypoventilation, apnea, dysrhythmias, hypotension and vasovagal episodes ${ }^{(3)}$.
Many studies have reported on use of propofol as a single agent for sedation during endoscopy. However propofol is often used in combination with another agent for other indications. The benefit of propofol in terms of shorter recovery and discharge times and higher patient satisfaction persists, when it is used in combination with other agents. Propofol has limited analgesic effect and higher doses are often required, when it is used as a single agent for colonoscopy, resulting in higher sedation levels. Thus use of propofol in combination with other agents may be preferable to propofol alone. The combination may be easier to manage due to lower sedation levels and ability to reverse some of the sedation with the use of reversal agents for narcotics and/or benzodiazepines ${ }^{(4)}$. 
Discomfort associated to colonoscopy results mainly from visceral nociception secondary to colonic distension and tractions. Yet, experimental studies demonstrate i.v. lidocaine is efficient in alleviating visceral pain. Accordingly, during visceral surgery, i.v. lidocaine allows for a $30-40 \%$ reduction in requirements of intraoperative volatile anaesthetics. I.V. lidocaine decreases also intraoperative propofol requirements during surgery under total i.v. anesthesia. These sparing effects are only observed during surgical stimulation, which suggests a property mediated by an antinociceptive action. These observations therefore extended to propofol PSA used to relieve visceral nociception during digestive endoscopy ${ }^{(5)}$.

\section{Aim of the work:}

Evaluation and comparing efficacy and safety of IV infusion of lidocaine versus magnesium sulfate as procedural sedation and analgesia (PSA) in colonoscopy.

\section{Patients and methods:}

After obtaining the local ethics committee of Minia University Hospital approval and written informed consent was taken from the patient, this prospective randomized double-blind controlled study was conducted in Minia University hospital during the period from August 2019 to March 2020, A total of 90 patients of both gender, American society of anesthesiologists (ASA) I and II, aged between 18-70 years old scheduled for colonoscopy with PSA, were included in the study.

\section{Exclusion criteria:}

- Using of general anesthesia,(with muscle relaxant and mechanical ventilator with endotracheal tube) or regional anesthesia.

- Patients with a history of allergy to any medical agent that used in the study.

- Pregnant and lactating females.

- Patients who refuse to participate in the study.

\section{Patient groups}

Patients were randomly allocated into 3 equal groups each containing 30 patients. Group (L) received $1.5 \mathrm{mg} / \mathrm{kg}$ of lidocaine IV before induction by propofol and continuous infusion of lidocaine $4 \mathrm{mg} \mathrm{kg} / \mathrm{h}$ after induction, group (M) received50 mg/ $\mathrm{kg}$ of magnesium sulfate before induction by propofol and continuous infusion of magnesium $8 \mathrm{mg} / \mathrm{kg} / \mathrm{h}$ after induction and group $(\mathrm{O})$ as control group received normal saline $0.9 \%$ Sodium Chloride. Anesthetic technique was standardized in both groups. Mean Arterial Blood Pressure, Heart rate, Respiratory rate, $\mathrm{O}_{2}$ saturation, episodes of desaturation, propofol requirements, postoperative pain, analgesic request and patient satisfaction were assessed.

\section{Results}

The two studied groups were comparable as regards the age, sex, weight, duration of operation with no significance as shown in table (1).

Table (1): Demographic data in the studied groups (data presented as mean \pm SD or number and percentage)

\begin{tabular}{|c|c|c|c|c|c|c|c|}
\hline \multicolumn{2}{|l|}{ Variables } & \multirow{3}{*}{$\begin{array}{c}\text { Group L } \\
\mathrm{N}=30 \\
25-67\end{array}$} & \multirow{2}{*}{$\begin{array}{c}\text { Group M } \\
\mathrm{N}=30\end{array}$} & \multirow{2}{*}{$\begin{array}{c}\text { Group O } \\
\mathrm{N}=30\end{array}$} & \multicolumn{3}{|c|}{ p-value } \\
\hline & & & & & $1 \& 2$ & $1 \& 3$ & $2 \& 3$ \\
\hline \multirow[t]{2}{*}{ Age/y } & Range & & $25-67$ & $19-67$ & \multicolumn{3}{|c|}{0.701} \\
\hline & Mean \pm SD & $44.9 \pm 13.8$ & $45.3 \pm 12.9$ & $42.4 \pm 16.6$ & 0.929 & 0.496 & 0.442 \\
\hline \multirow[t]{2}{*}{ Weight/Kg } & Range & $60-90$ & $60-90$ & $60-90$ & \multicolumn{3}{|c|}{0.984} \\
\hline & Mean \pm SD & $74 \pm 8.1$ & $74 \pm 8.1$ & $73.6 \pm 8.8$ & 1.000 & 0.878 & 0.878 \\
\hline \multicolumn{5}{|l|}{ Sex: } & \multicolumn{3}{|c|}{0.824} \\
\hline \multicolumn{2}{|l|}{ Males } & $18(60 \%)$ & $20(66.7 \%)$ & $20(66.7 \%)$ & \multirow[t]{2}{*}{0.833} & \multirow[t]{2}{*}{0.833} & \multirow[t]{2}{*}{1.000} \\
\hline \multicolumn{2}{|l|}{ Females } & $12(40 \%)$ & $10(33.3 \%)$ & $10(33.3 \%)$ & & & \\
\hline \multirow{2}{*}{\multicolumn{2}{|c|}{$\begin{array}{l}\text { Duration of } \\
\text { operation(min) }\end{array}$}} & \multirow[t]{2}{*}{$31.5 \pm 7.6$} & \multirow[t]{2}{*}{$31.5 \pm 7.6$} & \multirow[t]{2}{*}{$31.0 \pm 8.2$} & \multicolumn{3}{|c|}{0.955} \\
\hline & & & & & 1.000 & 0.794 & 0.973 \\
\hline
\end{tabular}

There was a significant increase in HR when comparing the 3 study groups and intragroup comparison as shown in table (2). 
MJMR, Vol. 31, No. 4, 2020, pages (95-100).

Table (2): changes in the heart rate (beat/min) (data presented as mean $\pm \mathrm{SD}$ )

\begin{tabular}{|c|c|c|c|c|c|c|c|c|c|}
\hline \multirow[t]{2}{*}{ Variables } & \multirow[t]{2}{*}{$\mathbf{N}$} & \multirow[t]{2}{*}{ Group L } & \multirow[t]{2}{*}{$\mathbf{N}$} & \multirow[t]{2}{*}{ Group M } & \multirow[t]{2}{*}{$\mathbf{N}$} & \multirow[t]{2}{*}{ Group O } & \multicolumn{3}{|c|}{ p-value } \\
\hline & & & & & & & $1 \& 2$ & $1 \& 3$ & $2 \& 3$ \\
\hline \multirow{2}{*}{ Basal HR } & \multirow[t]{2}{*}{30} & \multirow[t]{2}{*}{$88.4 \pm 8.7$} & \multirow[t]{2}{*}{30} & \multirow{2}{*}{$86.9 \pm 7.5$} & \multirow[t]{2}{*}{30} & \multirow{2}{*}{$89.3 \pm 10.2$} & \multicolumn{3}{|l|}{0.578} \\
\hline & & & & & & & 0.526 & 0.686 & 0.300 \\
\hline \multirow{2}{*}{$\begin{array}{l}\text { After } \\
\text { induction }\end{array}$} & \multirow[t]{2}{*}{30} & \multirow[t]{2}{*}{$73.2 \pm 5.6^{*}$} & \multirow[t]{2}{*}{30} & \multirow[t]{2}{*}{$73.0 \pm 5.9^{*}$} & \multirow[t]{2}{*}{30} & \multirow[t]{2}{*}{$82.8 \pm 10.1^{*}$} & \multicolumn{3}{|c|}{$<0.001$} \\
\hline & & & & & & & 0.905 & $<0.001$ & $<0.001$ \\
\hline \multirow[t]{2}{*}{$1 \mathrm{~min}$} & \multirow[t]{2}{*}{30} & \multirow[t]{2}{*}{$73.0 \pm 4.7^{*}$} & \multirow[t]{2}{*}{30} & $72.5 \pm 4.7^{*}$ & 30 & $79.2 \pm 8.1^{*}$ & $<0.00$ & & \\
\hline & & & & & & & 0.752 & $<0.001$ & $<0.001$ \\
\hline 2 min. & 30 & $72.9 \pm 5.0^{*}$ & 30 & $72.6 \pm 5.1^{*}$ & 30 & $79.1 \pm 7.4^{*}$ & $<0.00$ & & \\
\hline & & & & & & & 0.829 & $<0.001$ & $<0.001$ \\
\hline 3 min. & 30 & $73.1 \pm 5.4^{*}$ & 30 & $72.8 \pm 5.3^{*}$ & 30 & $79.3 \pm 8.0^{*}$ & $<0.00$ & & \\
\hline & & & & & & & 0.872 & $<0.001$ & $<0.001$ \\
\hline 4 min. & 30 & $73.4 \pm 4.9^{*}$ & 30 & $73.1 \pm 4.9^{*}$ & 30 & $79.2 \pm 7.0^{*}$ & $<0.00$ & & \\
\hline & & & & & & & 0.822 & $<0.001$ & $<0.001$ \\
\hline 5 min. & 30 & $73.9 \pm 5.0^{*}$ & 30 & $73.6 \pm 5.2^{*}$ & 30 & $79.2 \pm 7.0^{*}$ & $<0.00$ & & \\
\hline & & & & & & & 0.843 & 0.001 & $<0.001$ \\
\hline 10 min. & 30 & $74.4 \pm 4.8^{*}$ & 30 & $74.2 \pm 4.9^{*}$ & 30 & $79.9 \pm 7.4^{*}$ & $<0.00$ & & \\
\hline & & & & & & & 0.861 & 0.001 & $<0.001$ \\
\hline 15 min. & 30 & $73.8 \pm 4.7^{*}$ & 30 & $73.8 \pm 4.8^{*}$ & 30 & $79.5 \pm 7.2^{*}$ & $<0.00$ & & \\
\hline & & & & & & & 0.964 & $<0.001$ & $<0.001$ \\
\hline 20 min. & 30 & $74.2 \pm 4.7^{*}$ & 30 & $74.0 \pm 4.6^{*}$ & 28 & $80.6 \pm 6.5^{*}$ & $<0.00$ & & \\
\hline & & & & & & & 0.904 & $<0.001$ & $<0.001$ \\
\hline 25 min. & 26 & $74.4 \pm 5.1^{*}$ & 26 & $74.4 \pm 5.4^{*}$ & 24 & $80.5 \pm 7.8^{*}$ & 0.001 & & \\
\hline & & & & & & & 0.982 & 0.001 & 0.001 \\
\hline 30 min. & 16 & $76.5 \pm 5.2^{*}$ & 16 & $75.8 \pm 5.6^{*}$ & 16 & $82.8 \pm 6.3^{*}$ & 0.002 & & \\
\hline & & & & & & & 0.761 & 0.003 & 0.001 \\
\hline 35 min. & 12 & $75.1 \pm 4.5^{*}$ & 12 & $75.2 \pm 4.8^{*}$ & 12 & $82.6 \pm 5.3^{*}$ & 0.001 & & \\
\hline & & & & & & & 0.967 & 0.001 & 0.001 \\
\hline 40 min. & 10 & $75.2 \pm 4.7^{*}$ & 10 & $75.0 \pm 4.6^{*}$ & 8 & $81.6 \pm 6.5^{*}$ & $<0.00$ & & \\
\hline & & & & & & & 0.904 & $<0.001$ & $<0.001$ \\
\hline 45 min. & 2 & $74.8 \pm 4.7^{*}$ & 2 & $74.8 \pm 4.8^{*}$ & 2 & $80.5 \pm 7.2^{*}$ & $<0.00$ & & \\
\hline & & & & & & & 0.964 & $<0.001$ & $<0.001$ \\
\hline
\end{tabular}

There was a significant increase in MAP when comparing the 3 study groups and intragroup comparison as shown in table (3). 
Table (3): changes in the Systolic blood pressure (mmHg) (data presented as mean \pm SD).

\begin{tabular}{|c|c|c|c|c|c|c|c|c|c|}
\hline \multirow[t]{2}{*}{ Variables } & \multirow[t]{2}{*}{$\mathbf{N}$} & \multirow[t]{2}{*}{ Group L } & \multirow[t]{2}{*}{$\mathbf{N}$} & \multirow[t]{2}{*}{ Group M } & \multirow[t]{2}{*}{$\mathbf{N}$} & \multirow[t]{2}{*}{ Group O } & \multicolumn{3}{|c|}{ p-value } \\
\hline & & & & & & & $1 \& 2$ & $1 \& 3$ & $2 \& 3$ \\
\hline \multirow[t]{2}{*}{ Basal } & \multirow[t]{2}{*}{30} & \multirow[t]{2}{*}{$89.4 \pm 9.7$} & \multirow[t]{2}{*}{30} & \multirow[t]{2}{*}{$87.9 \pm 8.5$} & \multirow[t]{2}{*}{30} & \multirow[t]{2}{*}{$90.3 \pm 11.2$} & \multicolumn{3}{|l|}{0.578} \\
\hline & & & & & & & 0.526 & 0.686 & 0.300 \\
\hline \multirow{2}{*}{$\begin{array}{l}\text { After } \\
\text { induction }\end{array}$} & \multirow[t]{2}{*}{30} & \multirow[t]{2}{*}{$74.2 \pm 6.6^{*}$} & \multirow[t]{2}{*}{30} & \multirow[t]{2}{*}{$74.0 \pm 6.9^{*}$} & \multirow[t]{2}{*}{30} & \multirow[t]{2}{*}{$83.8 \pm 11.1^{*}$} & \multicolumn{3}{|c|}{\begin{tabular}{|l|}
$<0.001$ \\
\end{tabular}} \\
\hline & & & & & & & 0.905 & $<0.001$ & $<0.001$ \\
\hline \multirow[t]{2}{*}{$1 \mathrm{~min}}$. & \multirow[t]{2}{*}{30} & \multirow[t]{2}{*}{$74.0 \pm 5.7^{*}$} & \multirow[t]{2}{*}{30} & $73.5 \pm 5.7^{*}$ & 30 & $80.2 \pm 9.1^{*}$ & $<0.001$ & & \\
\hline & & & & & & & 0.752 & $<0.001$ & $<0.001$ \\
\hline 2 min. & 30 & $73.9 \pm 6.0^{*}$ & 30 & $73.6 \pm 6.1^{*}$ & 30 & $80.1 \pm 8.4^{*}$ & $<0.001$ & & \\
\hline & & & & & & & 0.829 & $<0.001$ & $<0.001$ \\
\hline $3 \mathrm{~min}$. & 30 & $74.1 \pm 6.4^{*}$ & 30 & $73.8 \pm 6.3^{*}$ & 30 & $80.3 \pm 9.0^{*}$ & $<0.001$ & & \\
\hline & & & & & & & 0.872 & $<0.001$ & $<0.001$ \\
\hline 4 min. & 30 & $74.4 \pm 5.9^{*}$ & 30 & $74.1 \pm 5.9^{*}$ & 30 & $80.2 \pm 8.0^{*}$ & $<0.001$ & & \\
\hline & & & & & & & 0.822 & $<0.001$ & $<0.001$ \\
\hline 5 min. & 30 & $74.9 \pm 6.0^{*}$ & 30 & $74.6 \pm 6.2^{*}$ & 30 & $80.3 \pm 8.0^{*}$ & $<0.001$ & & \\
\hline & & & & & & & 0.843 & 0.001 & $<0.001$ \\
\hline 10 min. & 30 & $75.4 \pm 5.8^{*}$ & 30 & $75.2 \pm 5.9^{*}$ & 30 & $80.9 \pm 8.4^{*}$ & $<0.001$ & & \\
\hline & & & & & & & 0.861 & 0.001 & $<0.001$ \\
\hline 15 min. & 30 & $74.8 \pm 5.7^{*}$ & 30 & $74.8 \pm 5.8^{*}$ & 30 & $80.5 \pm 8.2^{*}$ & $<0.001$ & & \\
\hline & & & & & & & 0.964 & $<0.001$ & $<0.001$ \\
\hline 20 min. & 30 & $75.2 \pm 5.7^{*}$ & 30 & $75.0 \pm 5.6^{*}$ & 28 & $81.6 \pm 7.5^{*}$ & $<0.001$ & & \\
\hline & & & & & & & 0.904 & $<0.001$ & $<0.001$ \\
\hline 25 min. & 26 & $75.3 \pm 5.5^{*}$ & 26 & $75.1 \pm 5.4^{*}$ & 24 & $81.5 \pm 7.4^{*}$ & $<0.001$ & & \\
\hline & & & & & & & 0.905 & $<0.001$ & $<0.001$ \\
\hline 30 min. & 16 & $73.9 \pm 5.0^{*}$ & 30 & $73.6 \pm 5.2^{*}$ & 30 & $79.2 \pm 7.0^{*}$ & $<0.001$ & & \\
\hline & & & & & & & 0.843 & 0.001 & $<0.001$ \\
\hline 35 min. & 12 & $74.4 \pm 4.8^{*}$ & 30 & $74.2 \pm 4.9^{*}$ & 30 & $79.9 \pm 7.4^{*}$ & $<0.001$ & & \\
\hline & & & & & & & 0.861 & 0.001 & $<0.001$ \\
\hline 40 min. & 10 & $73.8 \pm 4.7^{*}$ & 30 & $73.8 \pm 4.8^{*}$ & 30 & $79.5 \pm 7.2^{*}$ & $<0.001$ & & \\
\hline & & & & & & & 0.964 & $<0.001$ & $<0.001$ \\
\hline 45 min. & 2 & $74.2 \pm 4.7^{*}$ & 30 & $74.0 \pm 4.6^{*}$ & 28 & $80.6 \pm 6.5^{*}$ & $<0.001$ & & \\
\hline & & & & & & & 0.904 & $<0.001$ & $<0.001$ \\
\hline
\end{tabular}

There was no significant difference recorded when comparing the three studied groups as regard episodes of desaturations, as shown in (Table 4)

Table (4): Changes in episodes of desaturation $\left(\mathrm{SaO}_{2}\right)$ in percentage (data presented as mean $\pm \mathrm{SD}$ ).

\begin{tabular}{|l|l|l|l|l|l|l|}
\hline Variables & \multicolumn{1}{|c|}{ Group L } & \multicolumn{1}{c|}{ Group M } & \multicolumn{1}{|c|}{ Group O } & \multicolumn{3}{|c|}{ p-value } \\
\cline { 5 - 7 } & & & \multicolumn{1}{|c|}{$\mathbf{1 \& 2}$} & $\mathbf{1 \& 3}$ & $\mathbf{2 \& 3}$ \\
\hline No & $25(83.4 \%)$ & $23(76.6 \%)$ & $24(80 \%)$ & 0.745 & \\
Once & $4(13.3 \%)$ & $5(16.7 \%)$ & $4(13.3 \%)$ & 0.507 & 0.511 & 0.998 \\
Twice & $1(3.3 \%)$ & $2(6.7 \%)$ & $2(6,7 \%)$ & & & \\
\hline
\end{tabular}

There was a significant difference recorded when comparing propofol requirements in $\mathrm{mg}$ between the three study groups as shown in (table5) 
Table (5): Changes in propofol requirements in $\mathrm{mg}$

\begin{tabular}{|l|c|c|c|c|c|c|}
\hline Variables & Group 1 & Group 2 & Group 3 & \multicolumn{3}{|c|}{ p-value } \\
\cline { 5 - 7 } & & & & $\mathbf{1 \& 2}$ & $\mathbf{1 \& 3}$ & $\mathbf{2 \& 3}$ \\
\hline Mean \pm SD & $225.3 \pm 38.0^{*}$ & $354.0 \pm 49.1^{*}$ & $400.6 \pm 70.7^{*}$ & $<0.001$ \\
\cline { 4 - 6 } & & & & $<0.001$ & $<0.001$ & 0.001 \\
\hline
\end{tabular}

There was a significant difference recorded when comparing VAS between the three study groups as shown in (table6)

Table (6): Changes in VAS (data presented as mean $\pm \mathrm{SD}$ ).

\begin{tabular}{|c|c|c|c|c|c|c|}
\hline \multirow[t]{2}{*}{ Variables } & \multirow[t]{2}{*}{ Group L } & \multirow[t]{2}{*}{ Group M } & \multirow[t]{2}{*}{ Group 0} & \multicolumn{3}{|c|}{ p-value } \\
\hline & & & & $1 \& 2$ & $1 \& 3$ & $2 \& 3$ \\
\hline \multirow[t]{2}{*}{ After 15 min. } & \multirow[t]{2}{*}{$2.7 \pm 0.7$} & \multirow[t]{2}{*}{$2.9 \pm 0.7$} & \multirow[t]{2}{*}{$5.6 \pm 0.8$} & \multicolumn{3}{|c|}{$<0.001$} \\
\hline & & & & 0.252 & $<0.001$ & $<0.001$ \\
\hline \multirow[t]{2}{*}{ After 30 min. } & \multirow[t]{2}{*}{$1.0 \pm 0.6^{*}$} & \multirow[t]{2}{*}{$1.07 \pm 0.6^{*}$} & \multirow[t]{2}{*}{$3.8 \pm 0.6^{*}$} & \multicolumn{3}{|c|}{$<0.001$} \\
\hline & & & & 0.704 & $<0.001$ & $<0.001$ \\
\hline \multirow{2}{*}{ After 45 min. } & \multirow{2}{*}{$0.76 \pm 0.50^{*}$} & \multirow{2}{*}{$0.93 \pm 0.58^{*}$} & \multirow{2}{*}{$2.96 \pm 0.61^{*}$} & \multicolumn{3}{|c|}{$<0.001$} \\
\hline & & & & 0.260 & $<0.001$ & $<0.001$ \\
\hline \multirow[t]{2}{*}{ After 1 hour } & \multirow[t]{2}{*}{$0.36 \pm 0.49^{*}$} & \multirow[t]{2}{*}{$0.53 \pm 0.50^{*}$} & \multirow[t]{2}{*}{$2.33 \pm 0.66^{*}$} & \multicolumn{3}{|c|}{$<0.001$} \\
\hline & & & & 0.251 & $<0.001$ & $<0.001$ \\
\hline
\end{tabular}

There was a significant difference recorded when comparing patient satisfaction score between the three study groups as shown in (table7)

Table (7): Changes in patient satisfaction score (data presented as mean $\pm \mathrm{SD}$ ).

\begin{tabular}{|l|l|l|l|l|l|l|}
\hline Variables & Group L & Group M & Group O & \multicolumn{3}{|c|}{ p-value } \\
\cline { 5 - 7 } & & & & $\mathbf{1 \& 2}$ & $\mathbf{1 \& 3}$ & $\mathbf{2 \& 3}$ \\
\hline Mean \pm SD & $2.46 \pm .77$ & $2.36 \pm .80$ & $1.56 \pm .62$ & $<0.001$ & \\
\cline { 5 - 7 } & & & & 0.603 & $<0.001^{*}$ & $0.001^{*}$ \\
\hline
\end{tabular}

There was a significant difference recorded when comparing patient satisfaction score between the three study groups as shown in (table8)

Table (8): Changes in analgesic requirements (data presented as mean $\pm \mathrm{SD}$ ).

\begin{tabular}{|l|l|l|l|l|l|l|}
\hline Variables & \multicolumn{1}{c|}{ Group L } & Group M & \multicolumn{1}{|c|}{ Group O } & \multicolumn{3}{|c|}{ p-value } \\
\cline { 5 - 7 } & & & & $\mathbf{1 \& 2}$ & \multicolumn{1}{|c|}{$\mathbf{1 \& 3}$} & $\mathbf{2 \& 3}$ \\
\hline No & $26(86.7 \%)$ & $24(80 \%)$ & $3(10 \%)$ & $<0.001$ & \\
\cline { 5 - 8 } Yes & $4(13.3 \%)$ & $6(20 \%)$ & $27(90 \%)$ & 0.489 & $<0.001^{*}$ & $<0.001^{*}$ \\
\hline
\end{tabular}

\section{Discussion}

The clinical goals of administering GI endoscopy sedation are to relieve patient anxiety and discomfort, improve the outcome of the procedure, and decrease the memory of the event to the patient; so a number of different sedatives and analgesics may be used to achieve adequate sedation levels for endoscopic GI procedures ${ }^{(6)}$.

We found that the use of lidocaine infusion and magnesium sulfate infusion with propofol in patients undergoing colonoscopy under sedation decreases the total amount of propofol used, decreases post- 
procedural pain, also improves patient satisfaction, and decreases post-procedural analgesic demand and we also found that lidocaine is more effective than magnesium. Our results were in agreement with Forster et al., 2018 who assessed the effect of IV lidocaine infusion $(1.5 \mathrm{mg} \mathrm{kg}$ then 4 $\mathrm{mg} \backslash \mathrm{kg} \backslash \mathrm{h})$ in comparison to the control group on 40 patients scheduled for a colonoscopy under sedation with propofol and ketamine in a randomized placebocontrolled study and Khafagy et al., 2007 who compared two different doses of MgSo4; $50 \mathrm{mg} / \mathrm{kg}$ preoperatively followed by intravenous infusion of $8 \mathrm{mg} \backslash \mathrm{kg} \backslash \mathrm{h}$ or 16 $\mathrm{mg} \backslash \mathrm{kg} \backslash \mathrm{h}$ in 60 adults patients 25 to 60 years old undergoing herniorrhaphy under general anesthesia and found significant hemodynamic stability on the two MgSo4 groups in comparison to control group ${ }^{(7)}$.

\section{References}

1. Hinkelbein, Jochen; Lamperti, Massimo; Akeson, Jonas; Santos, Joao; Costa, Joao; De Robertis, Edoardo; Longrois, Dan; Novak-Jankovic, Vesna; Petrini, Flavia; Struys, Michel M.R.F.; Veyckemans, Francis; FuchsBuder, Thomas*; Fitzgerald, Robert $†$ European Society of Anaesthesiology and European Board of Anaesthesiology guidelines for procedural sedation and analgesia in adults, European Journal of Anaesthesiology: January 2018 - Volume 35 - Issue 1 - p 6-24
2. Rex DK, Khalfan HK. Sedation and the technical performance of colonoscopy. Gastrointest endoscopy Clin North America. 2005;15(4):661-72.

3. Dittmer, J. Colonoscopy and Timing of Sedation (Unpublished master's thesis). University of Calgary, Calgary, AB. 2016.

4. Singh H, Poluha W, Cheang M, Choptain N, Inegbu E, Baron K, Taback SP. Propofol for sedation during colonoscopy. Cochrane Database of Systematic Reviews 2008. .

5. FORSTER, C., et al. Intravenous infusion of lidocaine significantly reduces propofol dose for colonoscopy: a randomised placebo-controlled study. British journal of anesthesia, 2018, 121.5: 1059-1064.

6. Kahi CJ, Boland CR, Dominitz JA, Giardiello FM, Johnson DA, Kaltenbach T, Lieberman D, Levin TR, Robertson DJ, Rex DK: Colonoscopy surveillance after colorectal cancer resection: recommendations of the US multi-society task force on colorectal cancer. Am J Gastroenterol 2016.

7. KHAFAGY, Hanan F.; OSMAN, Emad S.; NAGUIB, Amr F. Effects of different dose regimens of magnesium on pharmacodynamics and anesthetic requirements of balanced general anesthesia. J Egypt Soc Parasitol, 2007, 37.2: 469-82. 